\title{
Botulinum Toxin Therapy for Squint
}

\author{
J. LEE, J. ELSTON, S. VICKERS, C. POWELL, J. KETLEY and C. HOGG \\ London
}

\begin{abstract}
Summary
Four hundred and five patients have been treated with injections of Botulinum neurotoxin A to extraocular muscles in the Botulinum Toxin Clinic at Moorfields Eye Hospital from November 1982 until the present. The indications and outcome of therapy are described and discussed.
\end{abstract}

In 1979, Alan Scott ${ }^{1}$ introduced the technique of injection of Botulinum neurotoxin $\mathrm{A}$ into extraocular muscles to correct squint. Although others such as Bach-y-Rita, ${ }^{2}$ Crone $^{3}$ and Jampolsky ${ }^{4}$ had suggested muscle injections with a variety of agents, Botulinum neurotoxin A proved to have a reliable reproducible effect in appropriate dosage, causing a temporary flaccid paralysis of the injected muscle lasting 8-12 weeks. The paralysis comes on about 3 days after injection, following which the deviation is reduced or even reversed due to unopposed action of the ipsilateral antagonist muscle. Although studies on mouse skeletal muscle by Duchen ${ }^{5,6}$ showed that injected muscles showed myofibrillar atrophy, muscular function seems to be regained fully as judged by clinical criteria of muscle power.

\section{Materials and Methods}

Having visited Alan Scott in San Francisco in 1981, we began treating patients for squint with Botulinum toxin in November 1982. From then until now we have treated 402 patients for squint and three for nystagmus. There were 227 female patients and 178 males. The youngest patient treated was 7 years old, the oldest was 87 years. The total number of injections given was 983 , giving an average of 2.42 injections per patient. Table I shows the total numbers of injections per patient and Table II shows the muscles injected on each treatment session.

All injections were given by intramuscular injection, with the toxin dissolved in sterile isotonic saline, in an injection volume of $0.1 \mathrm{ml}$. Initially bipolar injection electrodes were used, but the vast majority of injections were given with monopolar injection electrodes manufactured in the Electrodiagnostic Department at Moorfields Eye Hospital. Continuous electromyographic monitoring is performed during injection with both audible signal and oscilloscopic monitoring. Initially we used toxin manufactured by Dr Edward Schantz in Wisconsin and supplied to us by Alan Scott. Two hundred and ninety-eight injections were given of US-produced toxin in doses of $3.12 \times 10^{-4} \mu \mathrm{g}$ and $1.56 \times 10^{-3} \mu \mathrm{g}$. We then changed to toxin manufactured by Professor Melling's laboratory at Porton Down. To date, 695 injections have been given of the latter. As this formulation seems to have rather more biological activity, we use it in a dose of $6.25 \times 10^{-5} \mu \mathrm{g}$.

\section{Indications for Treatment}

(a) Concomitant Exotropia. This is the commonest indication for toxin therapy in

From the Botulinum Toxin Clinic Moorfields Eye Hospital, London.

Correspondence to: J. Lee, FRCS, Moorfields Eye Hospital, City Road, London EC1V 2PD. 
Table I.

\begin{tabular}{lrr}
\hline Number of injections & & \\
One injection & 165 & \\
Two injections & 115 & $(230)$ \\
Three injections & 56 & $(168)$ \\
Four injections & 27 & $(108)$ \\
Five injections & 11 & $(55)$ \\
Six injections & 9 & $(54)$ \\
Seven injections & 8 & $(56)$ \\
Eight injections & 1 & $(8)$ \\
Nine injections & 5 & $(45)$ \\
Ten injections & 4 & $(40)$ \\
Twelve injections & 2 & $(24)$ \\
Thirteen injections & 1 & $(13)$ \\
Seventeen injections & 1 & $(17)$ \\
\hline
\end{tabular}

Table II.

\begin{tabular}{r} 
Muscles injected per session \\
\hline LR-504 \\
MR-402 \\
IR-48 \\
Both MR- 13 \\
MR and LR- 9 \\
Both LR- 2 \\
LR and IO- 2 \\
MR and IR- 1 \\
IO- 1 \\
SR- 1 \\
-983
\end{tabular}

KEY: LR-Lateral rectus MR-Medial rectus IR-Inferior rectus SR-Superior rectus IO_-Inferior oblique

our clinic. A total of 183 patients have been treated (45.2 per cent of the total). Of these, seven had diplopia. The remaining 176 had either a primary or much more commonly, a consecutive exotropia, although 31 patients had a secondary sensory exotropia due to unilateral visual loss.

(b) Concomitant Esotropia. The total number treated was 102 (25.2 per cent). Five had diplopia, nine had a secondary esotropia in a blind eye and 88 had a primary or residual esotropia. A surprisingly large number of mature patients with esotropia had never had surgery because 'their parents did not believe in surgery'.

(c) Concomitant hypotropia. Three patients were treated for hypotropia (0.7 per cent), primarily for cosmetic reasons, although a secondary benefit was to assess the risk of post-operative diplopia.

(d) Dysthyroid Ophthalmopathy. We have treated 25 (6.2 per cent) patients with this condition. The preliminary results have been presented elsewhere ${ }^{7}$ and a more detailed study is in preparation. Twenty-one patients were treated for hypotropia by injections to the inferior rectus and four were treated for esotropia with injections to the medial rectus. Injection of the inferior rectus in dysthyroid disease is technically difficult and usually injections are given through the lower lid. In the majority of patients the deviation recurs, so the treatment is of main value in the control of acute diplopia in the early stages of the condition before surgery is indicated, although 6 patients seem to have been controlled to longterm single vision with toxin alone. The remarkable thing is that Botulinum toxin is as effective in this condition. Clearly, in the early stages of dysthyroid disease, the fibrotic changes we expect at surgery are not yet fully developed.

(e) Post-retinal Detachment Surgery. Twelve patients (3 per cent) were treated for diplopia following retinal detachment surgery. Botulinum toxin therapy has a particular advantage here in that it can be given without disturbing explants and causing extrusion.

(f) Orbital Trauma. Two patients with orbital trauma have been treated, without conspicuous success. One had a very extensive orbital crush injury with a blind eye, total ophthalmoplegia and a cosmetically poor exotropia: The other had a surgical overcorrection following freeing of a medial wall blow-out fracture.

(g) VI Nerve Palsy. A total of 49 patients with sixth nerve palsy have been treated (12.1 per cent). Of these, 34 were unilateral and 15 bilateral. The majority were post-traumatic following road traf- 
fic accident. We have actively sought patients with VI nerve palsy as we feel that Bøtulinum toxin has a particular role to play in management. Firstly, in partially recovered palsy, it may be possible to relax medial rectus contracture and permit the patient to regain binocular single vision. ${ }^{8}$ Further, if there is absolute lack of recovery, meaning that some form of muscle transposition is indicated, the medial rectus may be paralysed preoperatively, meaning that other muscles may be used to improve abduction without medial rectus tethering of the globe. The anterior segment circulation, however, runs much less risk of compromise, and there is an intact medial rectus muscle available for subsequent surgery if required. We are currently reviewing our experience with combined surgery and toxin therapy.

(h) Other Cranial Nerve Palsy. Six patients (1.5 per cent) have had treatment for other cranial nerve palsy, such as partial III nerve palsy and IV nerve palsy. Results are not quite as promising as in VI nerve palsy, but binocular single vision can be restored in suitable cases. Disappointingly, inferior oblique injection, which is technically very simple, is not very effective in superior oblique palsy.

(i) Decompensated Phoria. Patients whose control of a. large latent deviation (usually an exophoria) have been treated to reduce the deviation and improve control. Fifteen patients (3.7 per cent) have been treated and results have been very encouraging. In one patient, a 12 year old Asian girl, whose exophoria was just beginning to cause symptoms, one injection produced compensation of the deviation over a $3 \frac{1}{2}$ year follow-up period. One is tempted to wonder what the outcome would be of early treatment in intermittent exotropia.

(j) Surgical Overcorrection. Five patients were treated for surgical overcorrections, either where it was anticipated that the overcorrection would ultimately 'wear off', or where the surgeon preferred to avoid further surgery on already scarred muscles

(k) Convergence Deficit. Two patients had severe convergence insufficiency. In both, bilateral medial rectus resections had been of only temporary value. Botulinum toxin was given to a lateral rectus muscle in each case, but results were disappointing.

(1) Unilateral Aphakia. Four patients with acquired unilateral aphakia were treated to align the visual axes to see if fusion was possible with contact lens correction.

(m) Diagnostic. Quite early in this project, it became obvious that some patients with apparent suppression showed stereopsis and binocular function when the visual axes were aligned. Scott ${ }^{9}$ had pointed out the same phenomenon. Clearly, conventional orthoptic testing does not identify such patients. We have recently reviewed and presented our 'diagnostic' use of Botulinum toxin. ${ }^{10}$ The patients fall into two groups. (a) Those in whom there is a reasonable expectation of binocular single vision either because of the nature of the history or because of orthoptic tests. Here, the eyes are aligned and if BSV is present, surgery may be done. (b) This group is of patients wishing to have cosmetic improvement in whom there is a predicted risk of postoperative diplopia. The visual axes are aligned with injection. If the symptoms are bothersome, the effect is allowed to wear off and the patient is none the worse. If the patient is not subjectively distressed, however, more definitive surgery may be undertaken. Although other groups are also treated 'diagnostically', e.g. the unilateral aphakia group (above), there were 14 patients (3.5 per cent) in whom treatment was purely diagnostic, and we expect this proportion to increase.

(n) Nystagmus. Three patients have been treated for nystagmus and oscillopsia. The first was a 66 year old man with brainstem multiple sclerosis and oscillopsia. He had injection of medial and lateral rectus on the same eye and his acuity improved from $6 / 36$ to $6 / 18$. The effect was not maintained and reinjec- 
tion one month later gave little relief. The second was a 31 year old man with Freidreich's ataxia, confined to a wheelchair, with severe optic atrophy, gross horizontal nystagmus and oscillopsia. He has now had 7 treatments with injection of both medial and lateral rectus on one eye with subjective improvement of the oscillopsia. Injections need to be repeated at roughly 2 monthly intervals. The third patient was a 44 year old woman who had her right eye enucleated in infancy for retinoblastoma. From her earliest childhood she had been aware that she had oscillopsia in the primary position which improved if she adopted a head turn to the left and adducted the left eye. Kestenbaum surgery was suggested, but she was concerned about surgery on her only eye. Accordingly, she had a 'demonstration' in the form of an injection of Botulinum toxin to the left medial rectus. This produced the desired effect and she is soon to have surgery.

\section{Injections in Children}

We have little experience in treating children. Five have been treated under Ketamine anaesthesia. Of these, three have been effectively diagnostic, in that the aim was to demonstrate whether it was possible to re-align the visual axes. Two have since had surgery, one had a surgical overcorrection where the muscles were known to be very scarred, and toxin was preferred. One had an acquired VI palsy and was beginning to suppress and lose her compensatory head posture. We are dubious about the application of toxin therapy to infantile squint but it has been advocated by others. ${ }^{11}$

\section{Complications}

Fourty-three injections were followed by ptosis, usually partial. Nine patients had a subconjunctival haemorrhage after injection, and fifteen had affectation of a adjacent rectus muscle causing an acquired vertical deviation. The total of these was 67 , which gives a rate of 6.8 per cent, and all complications were transient. Many patients complained of temporary diplopia in the field of action of the injected muscle. This always resolved within a few weeks and patients were warned prior to injections of this.

\section{Outcome}

Ninety-three patients have had surgery performed. In 21 of these, the surgery was performed in conjunction with toxin pretreatment, as described under VI nerve palsy (above). Surgery has been recommended in 25 patients. In four patients, the initial deviation was treated with Botulinum toxin, then surgery was recommended. Following this, a consecutive deviation developed and this has been treated with Botulinum toxin to maintain good cosmesis. This group might be expected to increase with time. Seventy patients continue to attend the Toxin Clinic on a regular basis. A further 152 have been discharged, with advice to return in the event of deterioration of their current status. Fortyone patients have achieved a functional result, i.e., binocular vision has been restored in the primary position and over a reasonable field, and the situation is currently stable on follow-up of at least one year. Twenty patients have inadequate follow-up.

We would like to acknowledge the help of Mrs. Susan McKeever who prepared the manuscript.

\section{References}

${ }^{1}$ Scott AB: Botulinum toxin injection into extraocular muscles as an alternative to strabismus surgery. Ophthalmology 1979, 87: 1044-9.

${ }^{2}$ Bach-Y-Rita P: Personal communication, Quoted in Scott AB. Botulinum toxin injection of eye muscles to correct squint. Trans Am Ophthalmol Soc 1981, 79: 734-70.

${ }^{3}$ Crone R: Personal communication. Quoted in Scott AB. Botulinum toxin injection of eye muscles to correct squint. Trans Am Ophthalmol Soc 1981, 79: 734-70.

${ }^{4}$ Jampolsky A: Personal communication. Quoted in Scott AB. Botulinum toxin injection of eye muscles to correct squint. Trans Am Ophthalmol Soc 1981, 79: 734-70.

${ }^{5}$ Duchen LW: An electron microscopic study of the changes induced by Botulinum toxin injection in the motor end-plates of slow and fast skeletal muscle fibres in the mouse. J Neurol Sci 1971, 11: 47-60.

${ }^{6}$ Duchen LW: Changes in the electron microscopic structure of slow and fast skeletal muscle fibres in the mouse after local injection of Botulinum toxin. J Neurol Sci 1971, 14: 61-74.

${ }^{7}$ Lee JP and Elston JS: Treatment of acute dysthyroid ophthalmopathy with Botulinum Neurotoxin A. 
Presented at the 1985 meeting of the European Strabismologicla Association, Lausanne.

${ }^{8}$ Elston JS and Lee JP: Paralytic strabismus-the role of Botulinum toxin. Br J Ophthalmol 1985, 69: 891-6.

${ }^{9}$ Scott AB: Botulinum toxin injection of eye muscles to correct squint. Trans Am Ophthalmol Soc 1981, 79: $734-70$.
${ }^{10}$ Ketley MJ, Powell C, Lee JP, Elston JS: Botulinum toxin adaptation test: Use of Botulinum in the investigation of the sensory state in strabismus. Presented at the VIth International Orthoptic Congress, Harrogate; 1987.

11 Magoon E: Botulinum toxin chemo-denervation in infants and children. J Ped Ophthalmol and Strab 1986, 21: 110-12. 